\title{
(C) OPEN ACCESS \\ Prevalence and risk factors for diabetic retinopathy in China: a multi-hospital-based cross-sectional study
}

\author{
Guihua Zhang, Haoyu Chen, Weiqi Chen, Mingzhi Zhang
}

Department of Ophthalmology The Joint Shantou International Eye Center (JSIEC) of Shantou University and The Chinese University of Hong Kong, Shantou, Guangdong, China

\section{Correspondence to}

Dr Mingzhi Zhang, Department of Ophthalmology, The Joint

Shantou International Eye Center of Shantou University and The Chinese University of Hong Kong, Dongxia Road, Jingping District, Shantou 515041, Guangdong, China; zmz@jsiec.org

Received 9 February 2017 Revised 23 July 2017 Accepted 25 July 2017 Published Online First 30 August 2017

\section{ABSTRACT \\ Purpose To determine the prevalence and risk factors for diabetic retinopathy (DR) and sight-threatening diabetic retinopathy (STDR) in a multi-hospital-based DR screening programme among patients with diabetes in China, the Lifeline Express Diabetic Retinopathy Screening Program. \\ Methods Patients with diabetes in eight hospitals across mainland China (both southern and northern) from January 2014 to July 2016 were recruited in this programme. All participants underwent a standardised interview and examinations and received digital fundus photography. DR severity was graded from retinal fundus photographs by retina specialists in the reading centre of Joint Shantou International Eye Center, according to the grading standards of the English National Screening Programme. STDR was defined as the presence of preproliferative DR (R2), proliferative DR (R3) and/or maculopathy (M1).}

Results 16305 patients with diabetes were screened for DR in total. Fundus photographs were gradable for 15078 patients (92.5\%). The age-gender-standardised prevalence of any DR and STDR was $27.9 \%(95 \%$ $\mathrm{Cl}, 27.2 \%$ to $28.6 \%)$ and $12.6 \%(95 \% \mathrm{Cl}, 12.1 \%$ to $13.1 \%)$, respectively. In the multiple logistic regression analysis, younger age $(\mathrm{OR}, 0.967)$, longer duration of diabetes (OR, 1.093), higher haemoglobin A1c (OR, 1.115), higher fasting plasma glucose (OR, 1.074), higher systolic blood pressure (OR, 1.014), faster heart rate $(O R, 1.010)$, higher low-density lipoprotein $(O R$, 1.149), lower triglycerides $(O R, 0.975)$, higher blood urea nitrogen (BUN) $(O R, 1.012)$ and elevated serum creatinine level $(O R, 1.003)$ were associated with the presence of DR. Similar risk factors, except for BUN and triglycerides, were found for STDR.

Conclusions The prevalence of DR and STDR in diabetes was $27.9 \%$ and $12.6 \%$, respectively in this multi-hospital-based population across China. Compared with Western population with diabetes, similar risk factors for DR and STDR were found in Chinese patients with diabetes.

\section{INTRODUCTION}

Diabetic retinopathy (DR) is a leading cause of visual impairment and blindness in the working-age population across the globe. ${ }^{1}$ The number of people with diabetes mellitus (DM) is projected to approach 642 million people worldwide by 2040 .

It is estimated that $12 \%$ of adults have DM, and around half of Chinese adults have pre-diabetes. ${ }^{2}$ Several epidemiological studies have reported a high prevalence (ranging from $11.9 \%$ to $43.1 \%$ ) of DR and its risk factors among patients with DM in mainland China. ${ }^{3-5}$ However, these studies in China have showed differences in prevalence of and risk factors for DR because of their different study designs, grading standards and populations sampled. Screening for DR is accepted as an important means of early identification of the eyes with mild DR or sight-threatening diabetic retinopathy (STDR), which allows prompt intervention to prevent DR progression. ${ }^{6}$ In China, a national screening system for DR has not yet been established, and DR screening among diabetes has not been carried out in most places in China. The Lifeline Express Foundation launched the Diabetic Retinopathy Screening Program in multiple general hospitals across mainland China to screen diabetes, with an aim to reduce blindness in China by timely detection of DR and early treatment. On the other hand, the project also provides figures related to DR for government to make a strategy on diabetes healthcare. The aim of this study was to report the prevalence of DR and its risk factors under the Lifeline Express Diabetic Retinopathy Screening Program.

\section{SUBJECTS AND METHODS Subjects}

The Lifeline Express Diabetic Retinopathy Screening Program is a multi-hospital-based programme across mainland China. The current study used data from April 2014 to July 2016 from eight hospitals (Guilin City Second Hospital, Jilin City Chinese Traditional and Western Medicine Hospital, Jinan City Lixia District People's Hospital, Luoyang City Third Hospital, Luzhou City Red Cross Hospital, Huhehaote Neimengu Province People Hospital, Zhanjiang City Second Hospital, Zhengzhou City Second Hospital). The locations of the hospitals are shown in figure 1 . Before recruiting patients with $\mathrm{DM}$, a public information campaign on DR was carried out through the local newspaper, radio and television stations, as well as through posters in the outpatient hall of hospitals. DM was defined as random plasma glucose of $\geq 11.1 \mathrm{mmol} / \mathrm{L}$, fasting plasma glucose (FPG) of $\geq 7.0 \mathrm{mmol} / \mathrm{L}$, use of diabetes medication or a previous diagnosis of DM. ${ }^{7}$ All diagnosed subjects with DM were invited to participate in the programme free of charge. All participants registered at least 1 week in advance before screening. The participants were fully informed about the screening programme prior to registration. The study complied with the Declaration of Helsinki and was approved by the local hospital ethics committees. Written informed consent was obtained from each participant. 


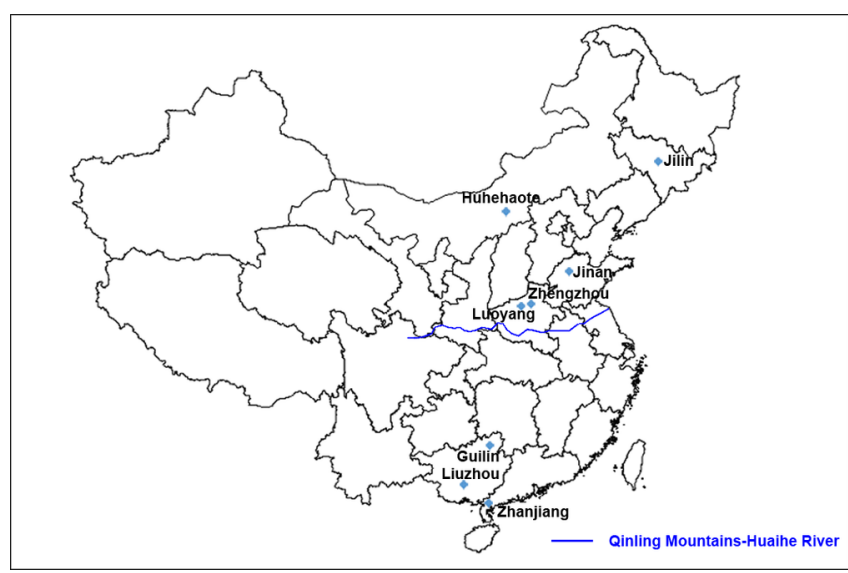

Figure 1 The locations of the hospitals.

\section{Screening procedure}

All the participants received a standardised interview (eg, date of birth, type of diabetes, duration of DM and treatment type) and a comprehensive clinical examination (eg, measurement of body weight, height, waist circumference, blood pressure and heart rate). Body mass index (BMI) was calculated by weight divided by the square of height in metres $\left(\mathrm{kg} / \mathrm{m}^{2}\right)$. Waist-to-hip ratio (WHR) was calculated using direct measurements. The blood samples of subjects were collected to check FPG, glycosylated haemoglobin (HbA1c), high-density lipoprotein (HDL), low-density lipoprotein (LDL), total cholesterol, triglycerides, serum creatinine and blood urea nitrogen (BUN).

Before pupil dilation, the habitual and pinhole visual acuity was measured using the ETDRS chart. Intraocular pressure in both eyes was measured with non-contact tonometer (CT-80; TOPCON, Tokyo, Japan and NT-4000; NIDEK, Gamagori, Japan). The anterior ocular segment was examined using slit lamp biomicroscopy (BQ900; Haag-Streit, Bern, Switzerland and SL-130; Carl Zeiss Meditech, Jena, Germany). Two $45^{\circ}$ fields (onemaculacentredandoneopticdisccentred) digital,colour, non-stereoscopic fundal photographs of each eye were taken using a non-mydriatic auto fundus camera (AFC-230; NIDEK, Gamagori, Japan or Canon CR-DGi retinal camera; Canon, Tokyo, Japan) after pupil dilation with tropicamide and phenylephrine eye drops (Santen Pharmaceutical, Osaka, Japan).

\section{DR grading procedure}

All the photographs were then sent to the reading centre of the Joint International Eye Center for DR grading. DR grading was performed according to the UK guidelines ${ }^{89}$ and through spatial resolution of $1024 \times 768$ pixels using a customised DR grading software named VisDR software with functions of magnification, adjustment of the luminance and the contrast, red free and statistical analysis. Retinal photographs were graded by trained retina specialists. Each photograph underwent primary grading. All photographs with any DR or any other eye disease and a randomly selected $15 \%$ of the photographs without any DR were submitted to a secondary grader for second grading. If there was any discrepancy between primary grading and second grading, the photograph was submitted to an arbitration grader (a senior ophthalmologist) for final decision. Quality assurance was ensured by recording sensitivity and specificity for each grader where sensitivity $\geq 95 \%$ and specificity $\geq 85 \%$ were considered acceptable. The graders underwent quarterly tests to ensure the accuracy of grading. STDR was defined as the presence of preproliferative DR (R2), proliferative DR (R3) and/
Table 1 Characteristics of subjects with diabetes in the Lifeline Express Diabetic Retinopathy Screening Program

\begin{tabular}{lcl}
\hline & Mean \pm SD & IQR \\
\hline Age (years) & $63.23 \pm 10.21$ & $57-70$ \\
\hline Duration of DM (years) & $7.45 \pm 6.42$ & $2.0-6.0$ \\
\hline HbA1c $(\%)$ & $7.48 \pm 1.78$ & $6.3-8.2$ \\
\hline FPG (mmol/L) & $8.10 \pm 2.58$ & $6.4-9.0$ \\
\hline Systolic blood pressure $(\mathrm{mm} \mathrm{Hg})$ & $133.44 \pm 16.18$ & $120-140$ \\
\hline Diastolic blood pressure $(\mathrm{mm} \mathrm{Hg})$ & $79.88 \pm 9.73$ & $73-85$ \\
\hline Height $(\mathrm{cm})$ & $161.92 \pm 8.23$ & $156-168$ \\
\hline Weight $(\mathrm{kg})$ & $64.13 \pm 11.58$ & $57-72$ \\
\hline BMI $\left(\mathrm{kg} / \mathrm{m}^{2}\right)$ & $24.75 \pm 3.41$ & $22.5-26.7$ \\
\hline Waist circumference $(\mathrm{cm})$ & $88.94 \pm 9.96$ & $82-96$ \\
\hline Hip circumference $(\mathrm{cm})$ & $95.18 \pm 9.59$ & $89-101$ \\
\hline WHR & $0.94 \pm 0.06$ & $0.89-0.98$ \\
\hline Heart rate & $77.32 \pm 9.93$ & $70-83$ \\
\hline Creatinine $(\mu \mathrm{mol} / \mathrm{L})$ & $74.93 \pm 35.00$ & $59-84$ \\
\hline BUN $(\mathrm{mmol} / \mathrm{L})$ & $5.70 \pm 4.76$ & $4.4-6.2$ \\
\hline HDL cholesterol $(\mathrm{mmol} / \mathrm{L})$ & $1.39 \pm 0.59$ & $1.04-1.56$ \\
\hline LDL cholesterol $(\mathrm{mmol} / \mathrm{L})$ & $2.70 \pm 0.99$ & $2.02-3.25$ \\
\hline Triglycerides $(\mathrm{mmol} / \mathrm{L})$ & $1.92 \pm 1.24$ & $1.18-2.30$ \\
\hline Total cholesterol $(\mathrm{mmol} / \mathrm{L})$ & $4.81 \pm 1.28$ & $4.01-5.61$ \\
\hline
\end{tabular}

BMI, body mass index; BUN, blood urea nitrogen; DM, diabetes mellitus; FPG, fasting plasma glucose; HbA1c, glycosylated haemoglobin; HDL, high-density lipoprotein; LDL, low-density lipoprotein; WHR, waist-to-hip ratio.

or maculopathy (M1). Photographs that were not assessable or could not be graded were considered ungradable (U). The DR grade for each subject was based on the worse DR grade of the two eyes. If one eye was non-STDR and the other eye was U, the subject would be graded as U. If one eye was STDR and the other eye $\mathrm{U}$, the subject would be classified as STDR.

\section{Statistical analysis}

IBM SPSS V.21.0 was used for statistical analyses. Agegender-standardised prevalence was calculated by using the 2010 population census of the People's Republic of China. The $\chi^{2}$ test for proportions and binomial distribution for directly computing CI were used. $\overline{\mathrm{x}} \pm \mathrm{s}$ was used to show means of continuous variables. Analysis of variance was used to compare means among multiple groups. Logistic regression models were applied to estimate the ORs and 95\% CIs for both any DR and STDR for all potential risk factors. The level of statistical significance was set at $\mathrm{p}<0.05$. The level of statistical significance was adjusted by Bonferroni correction when pairwise comparison in multiple groups was conducted.

\section{RESULTS}

In total, 16305 patients with DM were screened, including $1.07 \%$ with type 1 diabetes (175/16305), $98.39 \%$ with type 2 diabetes $(16043 / 16305)$ and $0.53 \%$ with pregnancy-related diabetes (87/16305). Fundus photographs were ungradable for 1227 (7.5\%) patients, which is in accordance with the UK standard of $<10 \%{ }^{10}$ The characteristics of subjects with diabetes are shown in table 1 . The age-standardised prevalence of any DR and STDR was $27.9 \%$ (95\% CI, 27.2\% to $28.6 \%)$ and $12.6 \%$ (95\% CI, $12.1 \%$ to $13.1 \%$ ), respectively. Among those, the age-gender-standardised prevalence of R1, R2, R3 and M1 was $22.1 \%$ (95\% CI, $21.4 \%$ to $22.8 \%$ ), $3.7 \%$ (95\% CI, $3.4 \%$ to 4.0\%), $2.1 \%$ (95\% CI, $1.9 \%$ to $2.3 \%$ ) and $11.2 \%$ (95\% CI, $10.7 \%$ to $11.7 \%)$, respectively. The prevalence of DR in hospitals ranged 
Table 2 Prevalence of diabetic retinopathy (DR) and sight-threatening diabetic retinopathy (STDR) in eight hospitals

\begin{tabular}{|c|c|c|c|c|c|c|c|}
\hline \multirow[b]{2}{*}{ Region } & \multicolumn{3}{|c|}{ DR } & \multirow[b]{2}{*}{$\begin{array}{l}\text { Age-gender- } \\
\text { standardised } \\
\text { prevalence (\%) }\end{array}$} & \multicolumn{3}{|l|}{ STDR } \\
\hline & $\begin{array}{l}\text { Number of subjects } \\
\text { with DM }\end{array}$ & $\begin{array}{l}\text { Number of subjects } \\
\text { with DR }\end{array}$ & $\begin{array}{l}\text { Crude prevalence } \\
(\%)\end{array}$ & & $\begin{array}{l}\text { Number of } \\
\text { subjects with } \\
\text { STDR }\end{array}$ & $\begin{array}{l}\text { Crude prevalence } \\
(\%)\end{array}$ & $\begin{array}{l}\text { Age-gender- } \\
\text { standardised } \\
\text { prevalence (\%) }\end{array}$ \\
\hline Southern region & 6531 & 1755 & 26.87 & 26.90 & 766 & 11.73 & 11.74 \\
\hline Luzhou & 2962 & 863 & 29.13 & 29.09 & 344 & 11.61 & 11.60 \\
\hline Zhanjiang & 1435 & 374 & 26.06 & 26.08 & 214 & 14.91 & 14.94 \\
\hline Guilin & 2134 & 518 & 24.27 & 25.19 & 208 & 9.74 & 9.73 \\
\hline Northern region & 8547 & 2449 & 28.65 & 28.84 & 1132 & 13.24 & 13.31 \\
\hline Jilin & 3489 & 804 & 23.04 & 23.05 & 295 & 8.46 & 8.45 \\
\hline Jinan & 2143 & 563 & 26.27 & 27.00 & 211 & 9.85 & 10.18 \\
\hline Luoyang & 1042 & 332 & 31.86 & 31.90 & 191 & 18.33 & 18.37 \\
\hline Huhehaote & 716 & 203 & 28.35 & 28.68 & 103 & 14.38 & 14.46 \\
\hline Zhengzhou & 1157 & 547 & 47.28 & 47.17 & 332 & 28.69 & 28.56 \\
\hline Total & 15078 & 4204 & 27.88 & 27.90 & 1897 & 12.58 & 12.63 \\
\hline
\end{tabular}

from $23.1 \%$ to $47.2 \%$, and that of STDR from $8.5 \%$ to $28.6 \%$. There was a significant difference in age-standardised prevalence of any DR $\left(\chi^{2}=204.34, \mathrm{p}<0.001\right)$ and STDR $\left(\chi^{2}=298.69, \mathrm{p}<0.001\right)$ between the eight hospitals (table 2).

Binary multiple logistic regression indicated that younger age (OR, $0.967 ; 95 \% \mathrm{CI} 0.961$ to 0.973 ), longer duration of DM (OR, 1.093; 95\% CI 1.084 to 1.103), higher HbA1c (OR, 1.115; $95 \%$ CI 1.078 to 1.154 ), higher FPG (OR, 1.074; 95\% CI 1.048 to 1.101), higher systolic blood pressure (OR, 1.014; 95\% CI 1.009 to 1.018$)$, faster heart rate (OR, $1.010 ; 95 \% \mathrm{CI} 1.004$ to 1.016), higher LDL (OR, 1.149; 95\% CI 1.079 to 1.224$)$, lower triglycerides (OR, 0.926 ; 95\% CI 0.874 to 0.982 ), higher BUN (OR, 1.012; 95\% CI 1.001 to 1.023$)$ and elevated serum creatinine level (OR, 1.003; 95\% CI 1.002 to 1.005) were associated with the presence of DR (table 3). Similar risk factors, except for WHR and triglycerides, were associated with STDR (table 4).

\section{DISCUSSION}

The overall age-gender-standardised prevalence of any DR and STDR was $27.9 \%$ and $12.6 \%$, respectively in our study.
However, the prevalence of any DR and STDR among eight hospitals showed significant differences. Risk factors for DR and STDR previously identified in other studies-longer diabetes duration, higher HbA1c and higher systolic blood pressurewere also identified in our study. ${ }^{4}{ }^{11-13}$

The prevalence of DR in our study was similar to that in other studies from Western countries (the Blue Mountains Eye Study in Australia and the Multi-ethnic Study of Atherosclerosis in the USA) ${ }^{14} 15$ and other Asian countries (the Singapore Indian Eye Study and the Singapore Malay Eye Study). ${ }^{12}{ }^{16}$ However, the prevalence of DR in China reported by studies published in the last 10 years showed large discrepancies, which ranged from $11.9 \%$ to $43.1 \%$. Hu et al reported that the prevalence of DR was only $11.9 \%$ in a population-based cross-sectional study in Liaoning Province, China. ${ }^{11}$ However in another investigation in rural China, the population-based Handan Eye Study found a prevalence of DR of $43.1 \%$. That figure was much higher than data reported in other studies from China, including our investigation. ${ }^{17}$ The discrepancy may be due to different study designs, grading standards and populations sampled. Our findings also

Table 3 Risk factors for any DR among patients with diabetes

\begin{tabular}{|c|c|c|c|c|}
\hline & \multicolumn{4}{|l|}{ Any DR } \\
\hline & Age-gender adjusted OR $(95 \% \mathrm{Cl})$ & $\mathrm{p}$ Value & Multivariate OR $(95 \% \mathrm{Cl})$ & p Value \\
\hline Age & $0.986(0.982$ to 0.989$)$ & 0.000 & $0.967(0.961$ to 0.973$)$ & 0.000 \\
\hline Gender & $0.939(0.874$ to 1.009$)$ & 0.086 & $1.023(0.915$ to 1.143$)$ & 0.695 \\
\hline Duration of DM (months) & $1.107(1.100$ to 1.113$)$ & 0.000 & $1.093(1.084$ to 1.103$)$ & 0.000 \\
\hline $\mathrm{HbA1c}(\%)$ & $1.205(1.180$ to 1.231$)$ & 0.000 & $1.115(1.078$ to 1.154$)$ & 0.000 \\
\hline FPG (mmol/L) & $1.126(1.110$ to 1.143$)$ & 0.000 & 1.074 (1.048 to 1.101$)$ & 0.000 \\
\hline Systolic blood pressure (mm Hg) & 1.015 (1.013 to 1.017$)$ & 0.000 & 1.014 (1.009 to 1.018$)$ & 0.000 \\
\hline Diastolic blood pressure $(\mathrm{mm} \mathrm{Hg})$ & $1.009(1.005$ to 1.012$)$ & 0.000 & $0.997(0.991$ to 1.004$)$ & 0.418 \\
\hline Heart rate & $1.015(1.011$ to 1.019$)$ & 0.000 & $1.010(1.004$ to 1.016$)$ & 0.001 \\
\hline $\mathrm{BMI}\left(\mathrm{kg} / \mathrm{m}^{2}\right)$ & $1.010(0.998$ to 1.023$)$ & 0.111 & $0.996(0.978$ to 1.016$)$ & 0.709 \\
\hline Waist circumference (cm) & $1.011(1.007$ to 1.016$)$ & 0.000 & $1.005(0.998$ to 1.012$)$ & 0.191 \\
\hline WHR & $1.101(0.542$ to 2.226$)$ & 0.790 & $0.521(0.192$ to 1.411$)$ & 0.200 \\
\hline Total cholesterol (mmol/L) & $1.067(1.031$ to 1.103$)$ & 0.000 & $0.972(0.924$ to 1.022$)$ & 0.266 \\
\hline Triglycerides (mmol/L) & $0.999(0.966$ to 1.034$)$ & 0.975 & $0.926(0.874$ to 0.982$)$ & 0.010 \\
\hline LDL cholesterol (mmol/L) & 1.141 (1.095 to 1.189$)$ & 0.000 & $1.149(1.079$ to 1.224$)$ & 0.000 \\
\hline HDL cholesterol (mmol/L) & $1.018(0.948$ to 1.094$)$ & 0.620 & $0.944(0.854$ to 1.044$)$ & 0.261 \\
\hline Creatinine $(\mu \mathrm{mol} / \mathrm{L})$ & 1.004 (1.003 to 1.005$)$ & 0.000 & $1.003(1.002$ to 1.005$)$ & 0.000 \\
\hline BUN (mmol/L) & $1.020(1.011$ to 1.030$)$ & 0.000 & $1.012(1.001$ to 1.023$)$ & 0.036 \\
\hline
\end{tabular}

BMI, body mass index; BUN, blood urea nitrogen; DM, diabetes mellitus; DR, diabetic retinopathy; FPG, fasting plasma glucose; HbA1c, glycosylated haemoglobin; HDL, highdensity lipoprotein; LDL, low-density lipoprotein; WHR, waist-to-hip ratio. 
Table 4 Risk factors for STDR among patients with diabetes

\begin{tabular}{|c|c|c|c|c|}
\hline & \multicolumn{2}{|l|}{ STDR } & \multicolumn{2}{|l|}{ DR } \\
\hline & Age-gender adjusted OR $(95 \% \mathrm{Cl})$ & $\mathrm{p}$ Value & Multivariate OR $(95 \% \mathrm{CI})$ & $\mathrm{p}$ Value \\
\hline Age & 0.983 (0.979 to 0.988$)$ & 0.000 & 0.959 (0.951 to 0.967$)$ & 0.000 \\
\hline Gender & 0.990 (0.898 to 1.191$)$ & 0.839 & 1.169 (0.996 to 1.371$)$ & 0.056 \\
\hline Duration of DM (years) & $1.100(1.092$ to 1.108$)$ & 0.000 & $1.097(1.084$ to 1.109$)$ & 0.000 \\
\hline $\mathrm{HbA1c}$ & $1.219(1.187$ to 1.251$)$ & 0.000 & $1.112(1.063$ to 1.163$)$ & 0.000 \\
\hline FPG (mmol/L) & 1.129 (1.110 to 1.149$)$ & 0.000 & $1.096(1.063$ to 1.131$)$ & 0.000 \\
\hline Systolic blood pressure $(\mathrm{mm} \mathrm{Hg})$ & $1.017(1.014$ to 1.020$)$ & 0.000 & $1.013(1.008$ to 1.019$)$ & 0.000 \\
\hline Diastolic blood pressure $(\mathrm{mm} \mathrm{Hg})$ & $1.010(1.005$ to 1.015$)$ & 0.000 & $0.994(0.985$ to 1.003$)$ & 0.211 \\
\hline Heart rate & $1.018(1.012$ to 1.024$)$ & 0.000 & $1.012(1.004$ to 1.020$)$ & 0.004 \\
\hline BMI $\left(\mathrm{kg} / \mathrm{m}^{2}\right)$ & $1.003(0.986$ to 1.020$)$ & 0.766 & 0.994 (0.968 to 1.021$)$ & 0.674 \\
\hline Waist circumference $(\mathrm{cm})$ & $1.013(1.007$ to 1.019$)$ & 0.000 & $1.005(0.995$ to 1.015$)$ & 0.349 \\
\hline WHR & $1.926(0.725$ to 5.116$)$ & 0.189 & $0.610(0.148$ to 2.524$)$ & 0.495 \\
\hline Total cholesterol (mmol/L) & 1.083 (1.034 to 1.135$)$ & 0.001 & 0.969 (0.902 to 1.041$)$ & 0.391 \\
\hline Triglycerides (mmol/L) & $1.020(0.973$ to 1.069$)$ & 0.408 & $0.947(0.889$ to 1.010$)$ & 0.096 \\
\hline LDL cholesterol (mmol/L) & $1.113(1.052$ to 1.178$)$ & 0.000 & 1.137 (1.039 to 1.244$)$ & 0.005 \\
\hline HDL cholesterol (mmol/L) & $1.028(0.931$ to 1.135$)$ & 0.588 & 0.965 (0.833 to 1.117$)$ & 0.630 \\
\hline Creatinine $(\mu \mathrm{mol} / \mathrm{L})$ & 1.007 (1.005 to 1.008$)$ & 0.000 & 1.006 (1.004 to 1.008$)$ & 0.000 \\
\hline BUN (mmol/L) & 1.023 (1.014 to 1.033$)$ & 0.000 & $1.013(1.000$ to 1.026$)$ & 0.053 \\
\hline
\end{tabular}

BMI, body mass index; BUN, blood urea nitrogen; DM, diabetes mellitus; FPG, fasting plasma glucose; HbA1c, glycosylated haemoglobin; HDL, high-density lipoprotein; LDL, lowdensity lipoprotein; STDR, sight-threatening diabetic retinopathy; WHR, waist-to-hip ratio.

highlighted that the prevalence in the Northern region $(28.7 \%)$ was higher than that in the Southern region (26.9\%). Similar results were reported by Liu et al in a meta-analysis of DR prevalence in mainland China. ${ }^{18}$ The risk factors were similar between the Southern region and the Northern region by respectively binary multiple logistic regression analysis. However, the mean duration of DM (8.91 years) in the Northern region is significantly longer than that ( 7.15 years) in the Southern region $(t=15.956, p<0.05)$. The discrepancy of the prevalence of DR between the Northern and Southern regions may mainly be caused by the difference in the duration of DM.

The overall prevalence of STDR was relatively high at $12.6 \%$ in our study. The high prevalence of STDR was mainly driven by the high prevalence of maculopathy. According to the UK guidelines, maculopathy is defined as having one or more of the following: exudates within one disc diameter (DD) of the centre of the fovea, circinate or groups of exudates within the macula, or any microaneurysms or haemorrhages within one DD of the centre of fovea if visual acuity $\leq 6 / 12 .{ }^{8}$ However, other studies such as the Beijing Eye Study and Singapore Eye Study had different definitions of maculopathy, for example, using the ETDRS guidelines. Moreover, only clinically significant macular oedema rather than macular oedema is included in STDR in these studies. It is therefore difficult to assert that the prevalence of STDR in our study was higher than that in other studies in mainland China, due to different grading systems. Comparing our results to the screening in Hong Kong which also followed the UK guidelines, the prevalence of maculopathy $(11.2 \%)$ in our study is higher than the findings (8.6\%) in Hong Kong. Relatively poor awareness and bad control of DM of participants in the mainland might be the reason. According to Xu et al's report, 7 out of 10 patients with diabetes are not aware of their high blood glucose, only $25.8 \%$ receive treatment for diabetes and only $39.7 \%$ of those treated have adequate glycaemic control in mainland China. ${ }^{19}$

In multivariate analysis, established risk factors for DR such as longer duration of diabetes, higher HbA1c level, hyperglycaemia and higher systolic blood pressure were confirmed in our study, suggesting acceptable validity of our results. Additionally, younger age, faster heart rate, higher LDL, lower triglycerides, higher BUN and higher serum creatinine were associated with DR in this study. The association of serum lipids with DR was obtained in this study, although the Multi-Ethnic Study of Atherosclerosis and the Singapore Indian Eye Study showed no associations of serum lipids with DR. ${ }^{12} 20$ These inconsistent findings imply that serum lipids are not strongly associated with DR. Similar to the Singapore Indian Eye Study and the Beijing Eye Study, DR was associated with younger age in our investigation. ${ }^{517}$ This association requires further exploration. In our study, we found that both BMI and WHR were not associated with DR. This association had been found in other DR investigations. ${ }^{1121}$ Although several previous studies including one study from China reported an association of BMI with DR presence, ${ }^{5} 162223$ we did not find this association in our study. Our finding implies that both WHR and BMI may not be good at predicting DR risk in China. We also observed that the presence of DR was associated with serum creatinine and heart rate. Moreover, this association was also found for STDR in our study. An association between serum creatinine and DR was reported previously by a national DR screening investigation from South Korea. ${ }^{24}$ The finding implied that renal function may play a certain role in DR progression. Two previous studies reported an association between DR and heart rate. ${ }^{25} 26$ However, the causes of faster heart rate can be physiological and pathological. In our screening, heart rate of each participant was not recorded in the same standard conditions. Thus, the association between heart rate and DR needs further exploration to confirm. Similar risk factors, except for BUN and triglycerides, were found for STDR in this study. The inconsistency in risk factors for DR and STDR implies that there are some other mechanisms for DR progressing to STDR.

The strengths of our study include the very large sample size compared with previous studies in China, the high proportion of gradable fundus pictures, the use of standardised grading protocols and the detailed assessment of risk factors. The limitations of our study should be mentioned. First, the cross-sectional design 
prevented direct conclusions on risk factors. Second, our maculopathy criteria may have overestimated the true prevalence of DM. The prevalence of DM cannot be directly compared therefore with that in other studies. Third, as a hospital-based study, the patients recruited into our study may not be representative of the overall population with diabetes. Finally, we did not include as variables other possible risk factors, such as family income, education and psychosocial factors.

In summary, our investigations have shown that approximately one-third of patients with DM in our large Chinese sample had DR, and more than 10\% had STDR. Similar risk factors for DR and STDR in Western populations with diabetes were found in Chinese patients with diabetes.

Acknowledgements The authors would like to thank Dr Michael Williams (Centre for Medical Education, Queen's University of Belfast, UK) and Professor Chi-Pui Pang (Department of Ophthalmology and Visual Sciences, The Chinese University of Hong Kong) for the critical comments and language polishing of the manuscript

Contributors GZ contributed to the design of the work, acquisition of data and analysis and interpretation of data; drafting the work and revising it critically for important intellectual content. $\mathrm{HC}$ contributed to the analysis and interpretation of data. WC critically revised the manuscript for important intellectual content. MZ made substantial contribution to the conception and design of the work; analysis and interpretation of data; drafting the work and revising it critically for important intellectual content.

Funding The programme was supported by Chinese Foundation for Lifeline Express and Lifeline Express Hong Kong Foundation.

Competing interests None declared.

Patient consent Obtained.

Ethics approval Hospital ethics committees.

Provenance and peer review Not commissioned; externally peer reviewed.

Data sharing statement The unpublished data are still being acquired for continuous screening programme. The data ownership belongs to the Chinese Foundation for Lifeline Express and Lifeline Express Hong Kong Foundation. The authors are authorised to use the data from the programme.

Open Access This is an Open Access article distributed in accordance with the Creative Commons Attribution Non Commercial (CC BY-NC 4.0) license, which permits others to distribute, remix, adapt, build upon this work non-commercially, and license their derivative works on different terms, provided the original work is properly cited and the use is non-commercial. See: http://creativecommons.org/ licenses/by-nc/4.0/

(c) Article author(s) (or their employer(s) unless otherwise stated in the text of the article) 2017. All rights reserved. No commercial use is permitted unless otherwise expressly granted.

\section{REFERENCES}

1 Cheung N, Mitchell P, Wong TY. Diabetic retinopathy. Lancet 2010;376:124-36.

2 Chan JC, Zhang Y, Ning G. Diabetes in China: a societal solution for a personal challenge. Lancet Diabetes Endocrinol 2014;2:969-79.

3 Pang C, Jia L, Jiang S, et al. Determination of diabetic retinopathy prevalence and associated risk factors in Chinese diabetic and pre-diabetic subjects: Shanghai diabetic complications study. Diabetes Metab Res Rev 2012;28:276-83.
4 Wang $\mathrm{FH}$, Liang $\mathrm{YB}$, Peng $\mathrm{XY}$, et al. Risk factors for diabetic retinopathy in a rural Chinese population with type 2 diabetes: the Handan Eye Study. Acta Ophthalmol 2011;89:e336-43.

$5 \mathrm{Xu}$ J, Wei WB, Yuan MX, et al. Prevalence and risk factors for diabetic retinopathy: the Beijing Communities Diabetes Study 6. Retina 2012;32:322-9.

6 Stefánsson E, Bek T, Porta M, et al. Screening and prevention of diabetic blindness. Acta Ophthalmol Scand 2000;78:374-85

7 American Diabetes A: diagnosis and classification of diabetes mellitus. Diabetes care 2014;37(Suppl 1):S81-90.

8 Harding S, Greenwood R, Aldington S, et al. Grading and disease management in national screening for diabetic retinopathy in England and Wales. Diabet Med 2003;20:965-71.

9 Lian JX, Gangwani RA, McGhee SM, et al. Systematic screening for diabetic retinopathy (DR) in Hong Kong: prevalence of DR and visual impairment among diabetic population. Br J Ophthalmol 2016;100:151-5.

10 Committee UNS. Essential elements in developing a diabetic retinopathy screening programme. Workbook version 4.4. UK: UK National Screening Committee. 2012 http://diabeticeyescreeningnhsuk/qualityassurance

11 Hu Y, Teng W, Liu L, et al. Prevalence and risk factors of diabetes and diabetic retinopathy in Liaoning province, China: a population-based cross-sectional study. PLoS One 2015;10:e0121477.

12 Zheng Y, Lamoureux EL, Lavanya R, et al. Prevalence and risk factors of diabetic retinopathy in migrant Indians in an urbanized society in Asia: the Singapore Indian eye study. Ophthalmology 2012;119:2119-24.

13 Ting DS, Cheung GC, Wong TY. Diabetic retinopathy: global prevalence, major risk factors, screening practices and public health challenges: a review. Clin Exp Ophthalmol 2016;44:260-77.

14 Wong TY, Klein R, Islam FM, et al. Diabetic retinopathy in a multi-ethnic cohort in the United States. Am J Ophthalmol 2006;141:446-55.

15 Mitchell P, Smith W, Wang JJ, et al. Prevalence of diabetic retinopathy in an older community. The Blue Mountains Eye Study. Ophthalmology 1998;105:406-11.

16 Wong TY, Cheung N, Tay WT, et al. Prevalence and risk factors for diabetic retinopathy: the Singapore Malay Eye Study. Ophthalmology 2008;115:1869-75.

17 Wang FH, Liang YB, Zhang $F_{\text {, et al. }}$. Prevalence of diabetic retinopathy in rural China: the Handan Eye Study. Ophthalmology 2009;116:461-7.

18 Liu L, Wu X, Liu L, et al. Prevalence of diabetic retinopathy in mainland China: a metaanalysis. PLoS One 2012;7:e45264.

$19 \mathrm{Xu}$ Y, Wang L, He J, et al. Prevalence and control of diabetes in Chinese adults. JAMA 2013:310:948-59.

20 Sasongko MB, Wong TY, Nguyen TT, et al. Serum apolipoprotein Al and B are stronger biomarkers of diabetic retinopathy than traditional lipids. Diabetes Care 2011:34:474-9.

21 Man RE, Sabanayagam C, Chiang PP, et al. Differential association of generalized and abdominal obesity with diabetic retinopathy in Asian patients with type 2 diabetes. JAMA Ophthalmol 2016:134:251-7.

22 Cheung N, Wong TY. Obesity and eye diseases. Surv Ophthalmol 2007:52:180-95.

23 Lim LS, Tai ES, Mitchell P, et al. C-reactive protein, body mass index, and diabetic retinopathy. Invest Ophthalmol Vis Sci 2010;51:4458-63.

24 Jee D, Lee WK, Kang S. Prevalence and risk factors for diabetic retinopathy: the Korea National Health and Nutrition Examination Survey 2008-2011. Invest Ophthalmol Vis Sci 2013:54:6827-33.

25 Imano E, Miyatsuka T, Motomura M, et al. Heart rate elevation and diabetic retinopathy in patients with type 2 diabetes mellitus and normoalbuminuria. Diabetes Res Clin Pract 2001;52:185-91.

26 Bulum T, Blaslov K, Duvnjak L. Resting heart rate is associated with nonproliferative retinopathy in normoalbuminuric type 1 diabetic patients. J Clin Hypertens 2013;15:579-83. 